muss daher den globalen Menschenrechtsdialog mit dem Ziel verstärken, die mentale Kluft zwischen aufgeklärter Moderne und fundamentalistischem Traditionalismus zu überbrücken und die Kultur der Intoleranz durch die Kultur der Toleranz zu ersetzen, damit sich die inneren Konflikte in diesen Gesellschaften nicht gewaltsam nach außen entladen.

Schwerpunkte deutscher Menschenrechtspolitik müssen neben der deutlichen Kritik an Menschenrechtsdefiziten u. a. in Tschetschenien und China der Einsatz gegen die Folter von Kindern, das Engagement für die Abschaffung der Todesstrafe, der Schutz der weltweit zunehmenden Zahl von Binnenvertriebenen, das Eintreten für die Einbeziehung von transnationalen Unternehmen in die Verantwortung für die Menschenrechte sowie die weltweite Verbesserung von Haftbedingungen sein.

Die Schaffung des Ständigen Internationalen Strafgerichtshofes für Kriegsverbrechen, Völkermord und Verbrechen gegen die Menschlichkeit ist ein wichtiger Baustein zum Aufbau einer weltweit verbindlichen Judikative zur Durchsetzung von Völkerrechtsnormen.

Außenpolitik und Wirtschaft müssen gemeinsam Wege zur Verstärkung der normativen, positiven und punitiven Ansätze zur Durchsetzung von Menschenrechten suchen. Das vielversprechende Modell einer OECD-Konvention gegen Korruption muss nun auch in die Tat umgesetzt werden.

Wolfgang Gehrcke

\title{
»Linke« Außenpolitik: Frieden und Gerechtigkeit
}

Wolfgang Gehrcke, MdB, Stell-
vertretender Vorsitzender und
außenpolitischer Sprecher der
PDS-Bundestagsfraktion, Ber-
lin

Michael Gorbatschow hatte als Generalsekretär der einst mächtigen Kommunistischen Partei der Sowjetunion das Ende des Kalten Krieges mit der Einsicht eingeläutet, dass die globalen Probleme der Menschheit globale Kooperation verlangten. $\mathrm{Zu}$ diesen Problemen rechnete er drohende Umweltkrisen, Unterentwicklung und Armut und nicht zuletzt die Gefahren des Wettrüstens. Mit dem schmählichen Untergang des großen Versuchs, der kapitalistischen Welt eine Alternative entgegenzusetzen, war das auf ideologischen Streit und militärische Überlegenheit fixierte Gegeneinander in der Weltpolitik am Ende angelangt. Der Weg schien frei, sich konstruktiv den gemeinsamen Überlebensfragen zuzuwenden.

Ein Fenster der Gelegenheit öffnete sich. Mit der Charta von Paris 1990 wurde eine Friedensordnung von Vancouver bis Wladiwostok skizziert, das Wort von der Friedensdividende machte die Runde. Mit den verschiedenen UNOGipfelkonferenzen zu Beginn dieses Jahrzehnts rückten Fragen einer gedeihlichen sozialen, wirtschaftlichen und ökologischen Weltentwicklung ins Zentrum des Interesses, wurden weitreichende Vereinbarungen zum Kampf gegen Hunger und Armut, gegen Umweltzerstörung und Unterdrückung erzielt. Der damalige UN-Generalsekretär Boutros-Ghali legte 1992 mit der Agenda für den Frieden ein umfassendes Konzept vor, wie die Vereinten Nationen ihre Schlüsselrolle bei der Entwicklung und Sicherung des Weltfriedens wahrnehmen sollten.

Es ist heute notwendig, sich dieser Prozesse zu erinnern. Dort wurden programmatische Grundlagen gelegt, auf denen aufgebaut werden kann: Sicherheit kann im globalen Maßstab nur noch gemeinsam, nicht mehr zu Lasten anderer erreicht werden; Frieden muss vor allem auf der Entwicklung einer sozial gerechteren, ökologisch bewussteren und demokratischer gestalteten Welt gründen; Stabilität kann nur durch verlässliche rechtliche Regularien erreicht werden - nicht durch militärische Überlegenheit.

\section{Globalisierung und Gewalt}

Tatsächlich brachten die neunziger Jahre eine weitreichende Zäsur. Doch die Weichen wurden in eine andere Richtung gestellt. Bestimmend für die globale Entwicklung war nicht Rio 1992, sondern Marrakesch 1994. Die Gründung der Welthandelsorganisation WTO symbolisierte, wie weit sich die Globalisierung unter den Vorzeichen neoliberaler Freihandelsdogmen institutionell verfestigt hatte. Bestimmend war nicht Paris 1990, sondern der Golfkrieg 1991 und die neue strategische Positionierung der NATO auf ihrem Gipfel von Rom (1991). Die »neue Weltordnung « des damaligen US-Präsidenten Bush sollte dem Worte nach zwar die Herrschaft des Rechts in den internationalen Beziehungen etablieren, beinhaltete aber im Kern das Recht militärischer Einmischungen durch die mit der »internationalen Gemeinschaft« identisch gesetzte westliche Staatenallianz. Das nach dem Ost-West-Gegensatz übriggebliebene Militärbündnis NATO gab sich die neue Aufgabe, ggf. weltweit Krisen »managen« zu wollen. Diese Entscheidung hatte unter anderem eine Konsequenz: Die Streitkräfte wurden auf Interventionsfähigkeiten »out of area« umgerüstet.

Der UNO wurden die nötigen Mittel zur Bewältigung der neuen Konflikte verweigert und somit erreicht, dass sie sich bei ihren Streitschlichtungsbemühungen von Mogadischu bis Srebrenica blamierte. Sie konnte anschließend an den Rand 
geschoben und durch von der übriggebliebenen Weltmacht USA dominierte Machtkartelle ersetzt werden.

Diese Entwicklung der letzten zwölf Jahre war begleitet von der Verheißung, die von der westlichen Allianz geführte Staatengemeinschaft werde dafür sorgen, dass die Welt friedlicher und stabiler werde. Doch trotz vordergründiger Erfolge, auf die die Protagonisten militärinterventionistischer Politik gerne verweisen (siehe Balkan-Region), muss konstatiert werden: Die Welt ist seitdem nicht friedlicher geworden, die Zahl der bewaffneten Konflikte hat zugenommen, die Spannungen in solch hochgerüsteten Regionen wie Nahost oder Südasien haben brisante Dimensionen erreicht.

Daraus wird nun umso mehr der Schluss gezogen, dass man sich dieser Entwicklung durch überlegene Streitkräfte und militärischem Eingreifen entgegenstellen müsse. Statt der Friedensdividende erleben wir seit vier Jahren die Trendwende zu steigenden Rüstungsausgaben. Mit dem neuen Interventionismus wird das zwischenstaatliche Gewaltverbot des Völkerrechts mehr und mehr ausgehoben.

Vorgeblich geht es um Menschenrechte, Demokratie und Frieden. Doch im Kern zielt der Vorsatz, Recht und Ordnung herstellen zu wollen, auf etwas anderes. Es geht darum, die Störpotentiale einer Globalisierung, die möglichst die ganze Welt den Imperativen des Marktes unterwerfen will, $\mathrm{zu}$ beseitigen. Störfaktoren können Flüchtlingsbewegungen ebenso sein wie das Auseinanderbrechen von Staaten, die Zunahme privatisierter Gewalt in den Krisenregionen oder widerborstige Regime in der randständigen Welt.

Die Paradoxie des neuen Interventionismus liegt nach meiner Überzeugung darin, dass die von den heutigen Machtstrukturen ausgehende Macht- und Interessenpolitik ständig aufs Neue die Probleme produziert, gegen die »weltpolizeilich« vorgegangen werden muss. Die neoliberale Globalisierung hat, indem sie die Macht der transnationalen Konzerne und des Finanzkapitals zu Lasten der Staaten immens gestärkt hat, Teile der Welt krisenanfälliger gemacht, zugleich aber die Möglichkeiten der Krisenregulation beeinträchtigt. Sie hat, indem sie den Graben zwischen reich und arm, mächtig und ohnmächtig weiter vertieft hat, maßgeblich dazu beigetragen, dass Gesellschaften vor dem Kollaps stehen, bewaffnete Gruppen ihr Heil in Plünderungen, Erpressungen und Terror suchen.

Dieser nur schwer zu übersehende Zusammenhang zwischen Globalisierung und Gewalteskalation muss auch für »linke« Außenpolitik gravierende Konsequenzen haben. Globalisierungskritik und Friedenspolitik gehören zusammen. Die katholischen Bischöfe hierzulande haben die Anforderung, die daraus erwächst, unter die schöne Formel des »gerechten Friedens « gebracht. Ohne nachhaltige Entwicklung, die dem größeren Teil der Menschheit erst eine Zukunftschance eröffnet, kann die Gewalt in der Welt nicht verringert werden. Ohne eine Friedens- und Sicherheitspolitik jedoch, die einen demokratischen Ordnungsrahmen für das Austragen der nötigen Konflikte schafft, wird Entwicklung weiter blockiert werden. Das Verhältnis der Staaten bzw. Staatengruppen untereinander auf verlässliche rechtliche Grundlagen zu stellen, das gesamte Potential diplomatischer Konfliktlösungen zu entwickeln, das sind für uns entscheidende Punkte bei der Zivilisierung der internationalen Beziehungen.

\section{Neue Herausforderung durch den internationalen Terrorismus}

Mit dem 11. September erreichte der internationale Terrorismus eine neue Dimension. Seine hemmungslose Gewalt, der Massenmord aus dem Innern der westlichen Gesellschaft heraus erzeugten Verunsicherung, vor allem aber die Furcht, Terroristen könnten in den Besitz von atomaren, chemischen oder biologischen Massenvernichtungswaffen gelangen. Dennoch meine ich, der 11. September war im eigentlichen Sinn des Wortes keine Zäsur. Terrorismus gibt es in der Geschichte schon lange, er ist immer an den gesellschaftlichen Kontext gebunden. Das legitimiert ihn selbstverständlich nicht. Es ändert aber auch nicht grundsätzlich das Bedingungsgefüge, in dem er auftritt. In seiner konkreten Gestalt ist er jeweils das Symptom für eine Krankheit, die aus den historisch-gesellschaftlichen Verhältnissen erwächst. Und in diesem Kontext muss er auch bekämpft werden. Die »amerikanische Antwort« aber, »das Übel des Terrorismus um jeden Preis auszumerzen $^{1}$, setzte in erster Linie auf militärische Gegenstrategien. Die Bildung einer weltweiten Koalition gegen den Terrorismus zeigte das amerikanische Interesse am engen (bilateralen) Schulterschluss mit möglichst vielen Partnern. In der Praxis stellte sich sehr schnell heraus, das sie vor allem der politischen Rückendeckung für ein in erster Linie militärisches Vorgehen dienen sollte.

Die Falken im Weißen Haus wollen die Gelegenheit nutzen, nicht nur Terroristen zu jagen, sondern auch missliebige Regimes zu beseitigen (»die Achse des Bösen «). Eine neue Doktrin macht die Runde, die »präemptive Militärschläge« gegen solche Regimes möglich machen soll. Diese Doktrin drängt konzeptionelle und materielle Möglichkeiten zur zivilen Bekämpfung des internationalen Terrorismus weitgehend in den Hintergrund. Das Handlungsschema ist einfach: Die USA führen im Wesentlichen mit modernen Kampfflugzeugen und anderen neuen Waffensystemen Militäraktionen durch. Mit Partnern, die sie zur Unterstützung dieser unilateralen Maßnahmen unbedingt benötigen, treffen sie bilaterale Absprachen. Nach den Militäraktionen wird den europäischen Verbündeten weitgehend die Aufgabe des Wiederaufbaus und die künftige Entwicklung der Region zugeschoben. Damit ist es seitens der USA völlig folgerichtig, die europäischen Konkurrenten in die verschiedenen Konfliktlagen dieser Welt einzubinden, schon allein weil dies erhebliche finanzielle, ökonomische, militärische, aber auch politisch-diplomatische Mittel der EU-Staaten bindet, die für bessere Zwecke fehlen.

\section{Falsche Antwort der deutschen Außenpolitik}

Schon die wenigen, nur holzschnittartig skizzierten Veränderungen in der Welt und in der internationalen Politik stellen auch die deutsche Außenpolitik vor neue Fragen, z. T. sehr grundsätzlicher Art. Ich nenne nur die Stichworte: Europäische Eigenständigkeit, Krieg als Mittel der Politik oder politische Gestaltung der Globalisierung. Niemand wird hier sofort Patentlösungen anbieten können, die es

1 Henry Kissinger, Die Herausforderung Amerikas. Weltpolitik im 21. Jahrhundert, München-Berlin 2002, S. 9. 
ohnehin nicht immergültig geben kann. Aber was ich an der offiziellen deutschen Außenpolitik kritisiere, ist, dass sie zum offensichtlich längst fälligen Paradigmenwechsel weder bereit noch fähig ist und sie damit das eine um das andere Mal die Chance für eine neue Politik verspielt! Mit dem Ende des Kalten Krieges und dem tatsächlichen Ende der Nachkriegszeit, die mit der Wiedervereinigung Deutschlands zusammenfielen, erhielt Deutschland seine volle Souveränität. Es war keine »ungebundene« Souveränität. Für Deutschland ergaben sich Verpflichtungen sowohl aus dem Grundgesetz wie dem allgemeinen Völkerrecht als auch aus dem Zwei-plus-Vier-Vertrag, der in seinem Artikel 2 festlegte, »dass von deutschem Boden nur Frieden ausgehen wird «. ${ }^{2}$ Aufgrund seiner ökonomischen Potenzen und seines wissenschaftlichen und kulturellen Leistungsvermögens, seiner historischen Verpflichtungen, seines politischen Gewichts und seiner Rolle in Europas Mitte war es geboten, sie in der internationalen Politik besonders verantwortungsvoll wahrzunehmen. Gerade von der rot-grünen Koalition hätte man erwarten können, dass sie das besonders sorgfältig tun und an den besten Seiten der Friedens- und Entspannungspolitik Willy Brandts anknüpfen würde. Brandt setzte auf zivile Lösung von Konflikten, auf Abrüstung und Beschränkung der Rüstungsexporte, die deutliche Erhöhung der Entwicklungshilfe für die ärmsten Länder, die Stärkung der UNO und die Erhöhung ihrer internationalen Rolle sowie die Einhaltung des internationalen Rechts. Seine Politik gründete sich auf die Ideen des Gewaltverzichts, der gemeinsamen Sicherheit und der Stärkung des Rechts.

Rot-Grün dagegen holte die Bundeswehr in das Arsenal gewöhnlicher außenpolitischer Instrumente zurück. Noch während des Kosovokrieges versuchte die Schröder-Regierung ihr völkerrechtswidriges Verhalten als absolute Ausnahme erscheinen zu lassen. Doch schon dieser Sündenfall trug entscheidend dazu bei, dass Deutschland wieder in den Krieg zog. Inzwischen sind wir bei der »Enttabuisierung des Militärischen« angelangt. Bundeskanzler Schröder erklärte im Lichte des 11. Septembers die »uneingeschränkte Solidarität mit den USA«. In seiner Regierungserklärung vom 11. Oktober 2001 postulierte er, Deutschland müsse sich »in einer neuen Weise der internationalen Verantwortung stellen $\ll^{3}$. Aktive Solidarität verpflichte zur Teilnahme an militärischen Operationen. Auslandseinsätze sind in der Tat zum Alltagsgeschäft der Bundeswehr geworden, der zur Feuerwehr- und Polizeiaktion umgedeutete Krieg gilt wieder als unumgängliches Mittel der Außenpolitik.

Wenn der Bundeskanzler der Bundesrepublik Deutschland nach den Terroranschlägen seine »uneingeschränkte Solidarität« mit den USA erklärt und zugleich deutlich macht, dass dies auch die uneingeschränkte Beteiligung an Militäreinsätzen einschließt, dann sollten wir den Kontext nicht aus den Augen verlieren: Die USA nehmen für sich inzwischen in Anspruch, ihnen nicht genehme oder gefährlich erscheinende Staaten mittels Krieg zu beseitigen. Damit würden die UNO und ihre größte Errungenschaft, das

2 Vertrag über die abschließende Regelung in Bezug auf Deutschland, in: Außenpolitik der Bundesrepublik Deutschland, Dokumente von 1949 bis 1994, Köln 1995, S. 700.

3 Deutscher Bundestag, 14. Wahlperiode, Plenarprotokoll der 192. Sitzung, Berlin, 11. Oktober 2001, S. 18682.
Gewaltverbot der Staaten, endgültig ad acta gelegt. Klüger wäre es, die Bundesregierung wirkte unter den westlichen Staaten darauf hin, das Gewaltmonopol der Vereinten Nationen $\mathrm{zu}$ festigen; klüger wäre es, die Bundesregierung engagierte sich aktiv im Rahmen der EU für nichtmilitärische Konzepte der Terrorismusbekämpfung und der Stabilisierung von Krisenregionen. Eine wichtige Voraussetzung für eine solche Politik wäre es, dass die Bundesregierung klarstellt, dass sie sich das undefinierte Freund-Feind-Schema, das die Staaten der Welt in gute und böse einteilt, nicht zu eigen macht.

Wir stehen zur gewachsenen internationalen Verantwortung Deutschlands. Sie wird am wirkungsvollsten wahrgenommen, wenn sich die Bundesrepublik Deutschland militär- und machtpolitisch zurückhält und zugleich ihre Beiträge für die Zivilisierung der internationalen Politik verstärkt.

\section{Nichtmilitärische Konfliktbewältigung statt Militärak- tionen und Krieg}

Der Bundestag hat in der vergangenen Wahlperiode siebzehn Mal Beschlüsse über Bundeswehreinsätze gefasst. Bundesregierung und die anderen Parteien verweisen gerne auf die Stabilitätsbemühungen, die Kriegseinsätzen gefolgt sind. Fakt ist: In keinem Fall haben die bisherigen Militäreinsätze eine dauerhafte Lösung gebracht, von den Kosten ganz abgesehen! Rein rechnerisch lässt sich beweisen: Hätte man nur einen Teil der für den Militäreinsatz aufgebrachten Mittel, von den Wiederaufbaukosten gar nicht zu reden, rechtzeitig und klug zur Lösung der nichtmilitärischen Belange eingesetzt, wäre die Situation in allen diesen Ländern heute stabiler, prosperierender. Und wie vielen Menschen wäre Flüchtlingselend und Not, ja gar der Tod erspart geblieben. Die Möglichkeiten der politischen, diplomatischen, ökonomischen und sozialen Lösungsansätze wurden in keinem der Fälle wirklich ausgeschöpft. Und in die gleiche Richtung weisen die Erfahrungen aus Afghanistan, wo die meisten Fakten noch der Öffentlichkeit vorenthalten werden.

Linke Politik setzt deshalb alles daran, dass das Motto »Vorbeugen ist besser als heilen« wieder ultimativen Charakter erhält! Priorität gebührt jedweder ziviler Prävention. Hier geht es im weitesten Sinne um die Förderung einer Kultur der Gewaltfreiheit, des interkulturellen Dialogs, der politischen Konfliktbewältigung, um die Stärkung des Rechts, die obligatorische friedliche Streitschlichtung, die Steigerung des Entwicklungshaushalts, die vollständige Abrüstung von Massenvernichtungsmitteln, die Beschränkung des Rüstungsexports, die Schaffung von Ausbildungsmöglichkeiten im Bereich ziviler Friedensdienste, die finanzielle Förderung der Friedensforschung, den Ausbau der Instrumente zur Durchsetzung von Wirtschaftssanktionen, die Schaffung internationaler Polizeikräfte und vieles mehr. Drohende Gewaltausbrüche müssen möglichst früh auf die internationale Tagesordnung gesetzt werden. Umso eher kann gewaltträchtigen Prozessen vorgebeugt und verhindert werden, dass sie in systematische Gewaltanwendung umschlagen.

Zugleich müssen frühzeitig die strukturellen Ursachen für Krisen und Konflikte sowie internationalen Terrorismus thematisiert werden und ihre Beseitigung angegangen werden. 
Ich nenne nur die ungleichen Machtverhältnisse in der Weltwirtschaft und die dadurch ausgelöste Polarisierung und Marginalisierung, globale Strukturprobleme. Und die Lösung der Konflikte muss sich vor allem auf soziale, ökologische, politische, rechtliche, kulturelle und humanitäre Komponenten stützen. Auch ist nachgewiesen, dass zivile Kräfte in der Vermittlung von Konflikten mehr erreichen als Militär, weil sie ohne eigenes Machtkalkül agieren. Außerdem sind sie weit »kostengünstiger«. Schon die »erste Welle« der militärischen Aktionen übersteigt in jedem Fall die Kosten für die nichtmilitärischen Gesamtanstrengungen um ein Vielfaches, von den »Kollateralschäden« abgesehen.

Aufgrund der Unterschiedlichkeit und der Komplexität der Krisen und Konfliktherde treten wir dafür ein, die verschiedensten Akteure - wirtschaftliche, politische, gesellschaftliche - und vor allem vermittelnde Kräfte aus den Konfliktregionen zur Problemregelung heranzuziehen und Anstrengungen sowohl regional wie global zu unternehmen. Die besten Voraussetzungen bieten dafür die UNO und die OSZE - vor allem wegen ihres Mitgliederkreises, ihres kooperativen Sicherheitsansatzes, aber auch wegen ihrer großen und ausdifferenzierten Konfliktverhütungskompetenz. Ohne Zweifel müssen beide dazu finanziell, personell und in ihren Kompetenzen weit mehr in die Lage versetzt werden. Schon wenn man jene Bereiche wirklich ausbauen und stärken würde, die es ihnen ermöglichen, unmittelbar und aus eigenen Kräften streitschlichtend und konfliktregelnd tätig zu werden, würde sich die Lage auf diesem Gebiet spürbar verändern. Wir sind aber auch dafür, bei der Konfliktbewältigung flexibel und kreativ einer dem jeweiligen Fall angepassten Arbeitsteilung $\mathrm{zu}$ folgen und sich gegenseitig ergänzende Bemühungen $\mathrm{zu}$ nutzen. Das gilt für die OAU, die Liga der arabischen Staaten, die Organisation der Islamischen Konferenz in Afrika, die ASEAN in Asien sowie die OAS auf dem amerikanischen Kontinent oder die OSZE und die EU in Europa wie viele andere Staatengruppen oder Organisationen, die im Zusammenwirken mit der UNO zu solchen Aktivitäten bereit sind.

Natürlich stellen auch wir in Rechnung, dass - wenn die Vermittlung erfolgt, also eine politische Lösung gefunden ist und z. B. ein Waffenstillstand abgeschlossen wurde - sich weder die streitenden Kräfte als auch ihre Waffen von einem Tag zum anderen in Luft auflösen. Ihre Auflösung bzw. das Einsammeln der Waffen, die internationale Absicherung des gefundenen Kompromisses könnte durch UN-BlauhelmKontingente, wie das aus der Vergangenheit nach Kapitel VI der UN-Charta bekannt ist, oder durch bei der UNO $\mathrm{zu}$ schaffende und von ihr einzusetzende internationale Polizeikontingente erfolgen.

Für besonders wichtig halten wir, ein produktiveres Verhältnis zwischen der großen und unentbehrlichen Arbeit der nichtstaatlichen Organisationen und der des Staates wie der UNO und der OSZE herzustellen. Das kommt zumeist der Soforthilfe für die besonders in Mitleidenschaft gezogene Zivilbevölkerung und der Entwicklungshilfe unmittelbar zugute. Die PDS redet nachdrücklich der Stärkung der Rolle der Frauen in der Konfliktbewältigung das Wort. Bei der Versöhnung verfeindeter Gruppen, beim Wiederaufbau der zivilen Gesellschaft leisten sie oft unersetzliche Beiträge.
Frauen gelingt es aufgrund ihrer sozialen Kompetenz besonders gut, Feindbilder abzubauen, Ethnien übergreifende Bündnisse einzugehen und überhaupt Konflikte konstruktiv auszutragen.

Entwicklungspolitik muss immanenter Teil der Friedenspolitik sein. Obwohl sich die deutsche Außenpolitik beinah in jeder »Sonntagsrede« zu diesem Grundsatz bekennt, hat sie einen schon mehr als 30 Jahre alten Beschluss der UNO, 0,7 Prozent des BIP für die Entwicklungszusammenarbeit bereitzustellen, bis heute nicht verwirklicht. Die Ankündigung der EU-Staaten von Monterrey, bis 2006 0,39 Prozent und bis 2015 endlich 0,7 Prozent des BIP bereitzustellen, sind kleine Schritte in die richtige Richtung, aber bei weitem nicht ausreichend. An Erklärungen und Zielen, ja auch Programmen fehlt es in der Regel nicht. Es ist vor allem eine Frage des politischen Willens. Schweden, die Niederlande, Dänemark und Luxemburg, Irland, Spanien und Großbritannien leisten seit vielen Jahren prozentual einen weitaus höheren Anteil. Deutschland muss sich ab sofort an diesen Beispielen orientieren und die Steigerung der Entwicklungsmittel auf eine gesetzliche Grundlage stellen. Das haben wir in dieser Legislaturperiode beantragt und werden dies auch in der nächsten tun.

Wir sind dafür, dass Deutschland eine Initiative für einen »Marshallpan für Entwicklung« ergreift, der alle Staaten zur Umschichtung von Rüstungsausgaben zur Entwicklungsförderung verpflichtet. In den internationalen Gremien sollte es vor allem dazu beitragen, globale politische und ökonomische Rahmenbedingungen zu schaffen, die es den Entwicklungsländern ermöglichen, ihre eigenen Märkte zu entwickeln und gleichzeitig Zugang zu den Märkten des Nordens zu erlangen. Für deutsche Politik muss die demokratische Kontrolle der internationalen Finanzströme und die Einbindung der internationalen Konzerne in die soziale Verantwortung ein wichtiges Ziel werden.

\section{Zivilmacht Europa}

Das Projekt »Europa«, dem sich einige weitsichtige Politiker nach zwei Weltkriegen widmeten, um nationalistische Todfeindschaften endgültig zu überwinden, ist mehr denn je aktuell. Europa wird gebraucht, um den Multilateralismus in der Welt zu stärken und die besseren Traditionen des Alten Kontinents fortzuentwickeln. Dazu gehören Kants Friedensethik wie der soziale Wohlfahrtsstaat gleichermaßen.

Dieses Projekt ist gleichzeitig mehr denn je fragil und gefährdet. Die nächsten Erweiterungsrunden stehen bevor. Die engere Assoziation der Gemeinschaft mit Russland, der Ukraine, den Ländern der Balkan-Region und der Türkei ist unabweisbar. Damit sind Grundsatzfragen der Verfassung dieses neuartigen politischen Konstrukts, nach seiner Funktionsfähigkeit und Entwicklungsrichtung aufgeworfen. Aber viel wichtiger noch: Die Art und Weise, wie der europäische Integrationsprozess von den bestimmenden Eliten bisher vorangetrieben wurde, gefährdet mehr und mehr die Integration selbst. Europaskeptische und europafeindliche Stimmungen und Strömungen verbreiten sich, rechtspopulistische Verführer haben derzeit europaweit Zulauf. Dass die Attacken gegen das Integrationsprojekt Resonanz finden, hat 
mit einem Europa zu tun, das bisher vor allem den Großbanken und transnationalen Unternehmen dient, einem Europa, das kein soziales Gesicht hat und das nicht demokratisch und durchsichtig verfasst ist.

Doch ein Zurück zum Europa der miteinander konkurrierenden Nationalstaaten, die Wiederkehr des »Hornviehnationalismus « (Peter Glotz, Nietzsche zitierend) würde einen Trümmerhaufen und unabsehbare Konsequenzen hinterlassen. Die PDS bejaht den europäischen Einigungsprozess. Wie vereinbart sich dies damit, dass sie bisher allen dem Bundestag vorgelegten Grundsatzentscheidungen über die Europäische Union die Zustimmung versagt hat? Sie hat die Verträge von Maastricht, Amsterdam, Nizza und die Einführung des Euro abgelehnt. Einzige Ausnahme: Das JA zur Grundrechtecharta. Ihr wird daher der Vorwurf gemacht, letztlich doch »europafeindlich « zu sein. Doch nichts falscher als das. Wir haben die Sorge - und fühlen uns durch die jüngste Entwicklung bestätigt -, dass der europäische Zug bisher in die falsche Richtung fährt. Die Weichen müssen neu gestellt werden, damit dieser Zug nicht entgleist. Mehr Wohlstand für alle, mehr statt weniger Sozialstaat, mehr Demokratie und Transparenz, nur so hat das europäische Projekt eine Chance. Unser Bestreben war es, die extreme soziale Schieflage des bisherigen Integrationsprozesses $\mathrm{zu}$ korrigieren und seine demokratischen Defizite zu beheben. Dabei bleibt es.

Wir befürworten die möglichst rasche Öffnung der EU für unsere mittel- und osteuropäischen Nachbarn. Dies bedingt, dass die inneren Strukturen der Gemeinschaft angepasst und weiterentwickelt werden. Es wird Aufgabe des Konvents sein, in dem wir aktiv mitarbeiten, diese schwierige Aufgabe innovativ zu meistern. Mit der neuen EU-Verfassung steht und fällt, ob die europäische Integration unumkehrbar gemacht werden kann.

Ich gehe noch einen Schritt weiter: Ich halte eine EU, die eine Rolle als weltpolitischer Akteur zu spielen versucht, für unverzichtbar. Daher ist auch das Bemühen, eine Gemeinsame Außen- und Sicherheitspolitik zu entwickeln, grundsätzlich unterstützenswert. Globale Stabilität kann nicht auf der Vorherrschaft eines einzelnen Staates gründen. Im Gegenteil. Wird die derzeitige Dominanz der USA fortgesetzt, droht die Welt weiter aus den Fugen zu geraten. Die EU wird - bei Wahrung der transatlantischen Partnerschaft die Aufgabe schultern müssen, ein Gegengewicht gegen diese Vorherrschaft zu bilden. Es geht um nicht mehr und nicht weniger als um die Emanzipation Europas. In diesem Prozess kommt es darauf an - um eine Wendung Egon Bahrs zu gebrauchen -, dass Europa seine Schwächen in Stärke verwandelt. Ein Wettrennen mit den USA auf dem Feld der harten Machtfaktoren, zum Beispiel der High-Tech-Rüstung, kann die EU nur verlieren. Stattdessen sollte sie sich auf das besinnen, was ihr Ansehen und Sympathie in der Welt eingetragen hat: diplomatische Klugheit, kooperative statt konfrontative Politik, solidarische Hilfe statt selbstherrlicher Arroganz, Handelsmacht statt Militärmacht.

Die EU sollte sich als weltpolitischer Akteur verstehen, der die Probleme des globalen Überlebens zum Ausgangspunkt seiner Politik macht. Dies heißt zugleich, dass die EU ihre Stärke vor allem in den Vereinten Nationen zur Geltung bringen muss. Sie kann in den internationalen Finanzinstitutionen die Einführung der Tobin-Tax voranbringen und dabei, wie ein wissenschaftliches Gutachten für das Bundesministerium für wirtschaftliche Zusammenarbeit gezeigt hat, auch mit gutem Beispiel vorangehen. Sie kann sich für eine Reform von IWF und Weltbank, für die Aufwertung des UN-Wirtschafts- und Sozialrats stark machen, damit dem neoliberalen Zerstörungswerk Einhalt geboten und eine nachhaltige Entwicklung des Südens eingeleitet werden kann.

Die EU tut gut daran, den außenpolitischen Fokus auf ihre Erweiterung Richtung Osten und Südosten und auf die Fortsetzung des Barcelona-Prozesses zu richten. Dies schließt das Bemühen um einen gerechten Frieden in Nahost ein.

Wir bleiben beim NEIN zu einer EU, die es den USA mit militärischer Machtprojektion gleich tun will. Und damit Nein zur Kriseninterventionstruppe, die ab 2003 einsatzbereit sein soll. Reformschritte, die es erleichtern, dass Europa in der internationalen Konfliktbearbeitung mit einer Stimme spricht, werden wir unterstützen. Aber das Konsensprinzip ist in diesem sensiblen Bereich für uns unverzichtbar. Es darf keine Lösungen geben, die den großen Staaten ein erdrückendes Übergewicht geben. Vor allem muss klar sein: Die Entscheidung über Krieg und Frieden ist nicht zu vergemeinschaften. Diese Verantwortung kann niemand den Einzelstaaten wegnehmen. In den Mitgliedsländern geht es freilich darum, die Möglichkeiten der parlamentarischen Kontrolle und der demokratischen Einflussnahme auszubauen und nicht einzuschränken, wie es leider alle Bundestagsfraktionen, Ausnahme PDS, in der nächsten Legislaturperiode vorhaben.

\section{Militärbündnisse auflösen - Kollektive Sicherheit}

Nach 1990 schien die Auflösung aller Militärbündnisse auf der Hand zu liegen. Doch die NATO, der der Feind abhanden gekommen war, suchte sich ein neues Betätigungsfeld. Sie schrieb sich die Aufgabe zu, Krisenintervention out-ofarea $\mathrm{zu}$ betreiben. Die Verteidigung des Bündnisgebietes wurde ersetzt durch die militärisch gestützte Verteidigung der Interessen der westlichen Allianz. Doch gut zehn Jahre nach dieser Umorientierung ist der weitere Weg der Allianz ungewiss. Erstmals in ihrer Geschichte hat die NATO nach den Terroranschlägen vom 11. September den Bündnisfall ausgerufen. In der Praxis blieb sie marginalisiert, da es die USA nach den komplizierten Erfahrungen des Kosovo-Krieges vorzogen, militärisch unilateral zu agieren. $\mathrm{Ob}$ die Initiativen von Generalsekretär Robertson, aber auch der Regierungschefs Blair und Aznar, der NATO im weltweiten Anti-Terrorkampf einen neuen Rang zukommen zu lassen, von Erfolg gekrönt sein werden, ist eher skeptisch $\mathrm{zu}$ sehen. $\mathrm{Ob}$ sie mit der zweiten Osterweiterung und dem neuen NATO-Russland-Rat »OSZE-isiert « wird, wie manche befürchten, kann niemand im Moment genau sagen. Aber zwei Residualfunktionen wird die Allianz mit großer Wahrscheinlichkeit haben:

- Die NATO bleibt eine »militärische Ressource«, auf die in wechselnder Konstellation bei Militäreinsätzen zurückgegriffen werden kann. 
- Sie bleibt ein Instrument zur Sicherung des politischen Zusammenhalts der westlichen Staatengemeinschaft unter der eindeutigen Führung der USA.

Gerade in letzterer Hinsicht sollte die für den Herbst geplante zweite Runde der Osterweiterung komplementär zur OstErweiterung der EU gesehen werden: Die Vereinigten Staaten trachten danach, damit ihren Einfluss in Mittel- und Osteuropa $\mathrm{zu}$ sichern.

Die PDS hat seit 1990 für die Auflösung aller Militärpakte plädiert. Wir sehen uns in der aktuellen Entwicklung bestätigt. Eine positive Rolle der NATO für Frieden und Sicherheit ist u.E. nicht zu erkennen. Täglich werden die zuständigen Minister durch die Allianz ermahnt, ihre Rüstungsanstrengungen zu intensivieren. Die NATO modernisiert ihre Waffen, statt für Abrüstung aktiv zu werden. Weil die NATO selbstmandatierte Militäreinsätze weiterhin für möglich hält, schwächt sie UNO und Völkerrecht. Dort wo sie begrenzte Erfolge einer Nachkriegsstabilisierung vorweisen kann, wie in der Balkan-Region, handelt es sich um Peace-keepingMissionen, die eigentlich der UN obliegen sollten. Offenkundig (miss-)braucht die Allianz diese Einsätze, um ihre Existenz weiter legitimieren zu können. Schließlich: Indem die NATO die US-Vorherrschaft konserviert und europäische Eigenständigkeit blockiert, schwächt sie zugleich die Entfaltung der Zivilmacht-Potentiale der EU.

Wir ziehen daraus den Schluss, dass die NATO ausgedient hat. Wir nehmen dabei zur Kenntnis, dass die NATO bei den Eliten des Westens, aber auch in relevanten Teilen der Öffentlichkeit symbolisch noch immer für die Verteidigung von Freiheit und Wohlstand steht. In Osteuropa drängt man immer noch danach, zu diesem exklusiven Club zu gehören. Diese internationale Konstellation, aber auch die gleichsam Staatsräson gewordene »Bündnistreue« der Bundesrepublik, führen dazu, dass ein einseitiger Austritt Deutschlands aus der NATO auf absehbare Zeit nicht auf der Tagesordnung steht.

Eine Bundesregierung, die ernsthaft auf Zivilisierung und Entmilitarisierung setzen würde, hätte aber Möglichkeiten, ihren Einfluss innerhalb der Allianz zur Geltung zu bringen. Sie hätte unseres Erachtens die Aufgabe, innerhalb der NATO die nukleare Erstschlagsoption permanent in Frage zu stellen (gerade nach den neuesten Entwicklungen in den USA); sie müsste sich dem neuen Rüstungswettlauf widersetzen und stattdessen Abrüstungsvorschläge unterbreiten; schließlich sollte sie öffentliche Debatten über die im Washingtoner Dokument von 1999 niedergelegte Einsatzdoktrin nicht scheuen.

Zugleich geht es darum, über eine zukünftige europäische Friedensordnung nachzudenken. Wir werben dafür, die NATO durch ein kollektives Sicherheitssystem im euro-atlantischen Raum zu ersetzen. Ob dafür die OSZE der geeignete Organisationsrahmen ist, erscheint heute überaus fraglich. Die OSZE, die nach 1990 eine kurze Blütezeit erlebte, ist inzwischen einem schleichenden Auszehrungsprozess ausgesetzt. Sie fungiert als Subunternehmen - ihr werden lediglich Aufgaben wie Wahlbeobachtung oder die Hilfe beim Aufbau von »Zivilgesellschaften« übertragen. Gegen die Vorstellung, die OSZE in eine zentrale Rolle zu bringen, wird gern eingewandt, selbst Russland habe sein früheres Interesse an dieser Organisation verloren. Wahr ist, dass Russland bislang nicht bereit ist, in jedem Falle die »guten Dienste« der OSZE in Krisen und Konflikten auf seinem Territorium in Anspruch zu nehmen, weil sie instrumentalisiert würden. Das mag stimmen oder nicht. Der Westen hat kein Recht, solange er selbst anders verfährt, Russlands Interesse an der OSZE und seine Glaubwürdigkeit in Frage zu stellen. Wir vertreten den Ansatz: Eine OSZE-Vermittlerrolle muss in allen entsprechenden Konflikten möglich sein, d. h. von Nordirland über das Baskenland bis nach Mittelasien. Es darf sich nicht der Eindruck verfestigen, dass ein Menschenrechte verletzender Staat immer dann mit Samthandschuhen behandelt wird, wenn er der NATO angehört oder den USA zu Diensten ist.

Nach Lage der Dinge ist die OSZE heute die einzige Organisation, die die nötigen universellen Eigenschaften für eine euro-atlantische Sicherheitsarchitektur mitbringt. Und sie verfügt über ein Instrumentarium zur Krisenprävention und zur nichtmilitärischen Konfliktbewältigung. Sie hat mit ihren Institutionen auf dem Balkan, im Kaukasus, in Zentralasien und in anderen Konfliktregionen keine schlechte Arbeit geleistet. Ihr Beitrag könnte größer sein, wenn man sie denn ließe.

Es wird aber Zeit brauchen, um diese Organisation in eine neue, politische Schlüsselfunktion zu bringen. Die Bundesrepublik Deutschland müsste dazu beginnen, den von den verschiedenen Bundesregierungen erklärten Politikansatz »OSZE First « konsequent umzusetzen. Die OSZE und ihre Einrichtungen sollten aktiver bei der Konfliktprävention eingeschaltet und entsprechend gestärkt werden. Dazu wäre es von Nutzen, wenn die EU ihre im Aufbau befindlichen Instrumente der Krisenfrühwarnung (u. a. Satellitenaufklärung) und der zivilen Streitbeilegung in den OSZE-Handlungsrahmen einbringen würde. Die OSZE muss aufgewertet werden, indem in ihrem Rahmen neue Initiativen für Abrüstung und Rüstungskontrolle entwickelt werden. Uns kommt es auf die Grundprinzipien dieser Sicherheitsarchitektur an; der passende institutionelle Rahmen bedarf selbstverständlich entsprechender internationaler Übereinkünfte.

\section{Abrüstung als positiver Wert}

Abrüstungspolitik ist aus der Mode geraten. Zwar wurde in jüngster Zeit mit großem Tamtam der Durchbruch bei den Nukleararsenalen der USA und Russlands gefeiert, aber die begrüßenswerte Vereinbarung zwischen Bush und Putin hat so viele Schwachstellen, dass kein Anlass besteht, sich beruhigt zurückzulegen. Ansonsten stagnieren die verschiedenen Abrüstungsforen, während die rüstungstechnologische Modernisierung Fortschritte macht. Auch die jetzige Bundesregierung schreibt Abrüstung klein, ist bestenfalls rüstungskontrollpolitisch engagiert. Für uns ist Rüstungskontrolle auch nach dem Ost-West-Konflikt ein nützliches Instrument der Einhegung destabilisierender Entwicklungen und der internationalen Vertrauensbildung. Daher unterstützen wir das Bemühen um ein Verifikationsprotokoll bei den biologischen Waffen oder die Fortsetzung des open-skies-Regimes. Aber wir wollen vor allem echte Fortschritte bei der Reduzierung der Waffenpotentiale, der Streitkräfte und der Rüstungsausgaben. Abrüstung hat für uns weiterhin einen eigenen Stellenwert. Jeder Euro, der für eine Rakete ausgegeben wird, kann 
nicht in Bildungs- oder Entwicklungsprogramme investiert werden. Tötungsinstrumente $\mathrm{zu}$ entwickeln, $\mathrm{zu}$ bauen, $\mathrm{zu}$ vertreiben und sie einzusetzen, ist für uns nicht zuletzt eines: eine Vergeudung von Ressourcen. Nur verteidigungspolitische Notlagen können solche »Fehlinvestitionen« rechtfertigen. Diese Gründe können nicht mehr geltend gemacht werden. Da Abrüstung auf der internationalen Agenda nicht obenan steht, sollte die Bundesrepublik alle Spielräume für einseitige Schritte nutzen. Wir haben eine erhebliche Verringerung der Bundeswehr, die Aufhebung der Wehrpflicht und ein Zurück zum Verteidigungsauftrag vorgeschlagen. Wir wollen die großen Entwicklungs- und Beschaffungsprogramme, die in den nächsten Jahren Summen im dreistelligen Milliardenbereich verschlingen werden, drosseln, bzw. nach Möglichkeit ganz stoppen.

Eine radikale Rüstungsminderung kann weder in Washington noch bei der NATO in Brüssel auf Wohlgefallen hoffen. Aber der für diesen Fall in der sicherheitspolitischen Fachwelt gerne heraufbeschworene Einflussverlust Deutschlands würde durch die wirtschaftliche und politisch-moralische Stärkung aufgewogen. Wir haben vorgeschlagen, die durch Abrüstung freiwerdenden Mittel für Konversion und Entwicklungszusammenarbeit zu verwenden. Damit könnte Deutschlands Ansehen gerade in den Ländern des Südens beträchtlich erhöht werden. Dieses Gewicht trägt auf lange Sicht mehr, als Einsatztruppenversorger oder Abstandswaffen.

Wir plädieren dafür, bei den Rüstungsexporten die Anfang 2001 verabschiedeten Politischen Grundsätze und die dort verankerten Kriterien - Menschenrechte, Entwicklung, Konfliktentschärfung - ernst zu nehmen. Deutschland sollte noch restriktiver bei Rüstungsgeschäften verfahren. Allein in der Einschränkung der Ausfuhr oder Lizenzvergabe bei den Kleinwaffen hätte die Bundesrepublik ein reichhaltiges Betätigungsfeld.

Abrüstung ist zugleich das wirksamste Mittel gegen die drohende bzw. akute Gefahr einer Weiterverbreitung von Massenvernichtungswaffen. Fakt ist, dass ungeachtet der viele Jahrzehnte während des Ost-West-Konflikts erfolgreichen Politik der Nichtweiterverbreitung von Atomwaffen und des Verbots anderer Massenvernichtungswaffen weitere Staaten solche Waffen entwickelt haben. Und niemand kann mit absoluter Sicherheit ausschließen, dass sich internationale Terroristen Massenvernichtungswaffen aneignen. Die Wahrheit gebietet aber festzustellen, dass dies in vielem der Politik der jetzigen Nuklearmächte geschuldet ist. Sie haben die versprochene atomare Abrüstung nicht vorangetrieben, wollten sie doch einerseits die Atomwaffen behalten und immer präzisere produzieren, andererseits andere Staaten daran hindern, solche Waffen herzustellen. Im Prinzip das gleiche Spiel erleben wir mit dem Atomteststoppvertrag oder dem Verbot der Chemiebzw. biologischen Waffen.

Wir lehnen es entschieden ab, dass man den Einsatz dieser Waffen gegen solche Staaten, denen man den Bau dieser Waffen zutraut bzw. unterstellt, wie in US-amerikanischen Militärplanungen jüngst geschehen, auch nur in Erwägung zieht. Grundsätzlich wird es keine friedliche Welt geben, in der die einen Staaten für immer auf diese Waffen verzichten, während andere, die zumeist auch über überlegene konventionelle militärische Fähigkeiten verfügen, sie zur Durchsetzung politischer Ziele nutzen können. Es gibt nur einen Ausweg:
Fortsetzung der nuklearen Abrüstung bis hin zu einer atomwaffenfreien Welt. Und was für die Atomwaffen gilt, trifft im Prinzip auch auf die biologischen und die chemischen Waffen $\mathrm{zu}$.

Auch aus diesen Erwägungen heraus lehnen wir den gegenwärtig von der Bush-Administration vorbereiteten Krieg gegen den Irak ab. Ein solch massiver Streitkräfteeinsatz - die Rede ist von etwa 200.000 Soldaten der Land- und Luftstreitkräfte sowie 700 bis 1.000 Flugzeugen - wäre ein eklatanter Bruch des Völkerrechts. Selbst wenn die Behauptung stimmen würde, dass sich das dortige Regime um Massenvernichtungswaffen bemühe (was ja in der Tat nicht auszuschließen ist), müsste nach anderen Wegen gesucht werden, um die Gefahr zu bannen. Die Rückkehr der Waffeninspekteure ist dabei ein probates Mittel. Im Gegenzug wären die brutalen Sanktionen gegen den Irak zu lockern.

Die Bundesregierung weicht bisher dieser Auseinandersetzung aus und hofft, dass der Kelch der Kriegsbeteiligung an ihr vorübergehen möge. Andere drängen aufs Mitmachen. Die PDS verfolgt seit Jahren einen anderen Ansatz: Der Irak muss schrittweise und konditioniert in die internationale Gemeinschaft aufgenommen werden. Es muss endlich über ein regionales Sicherheitssystem und die Weiterführung der Abrüstung in dieser Region entsprechend den einschlägigen UN-Resolutionen gesprochen werden. Geeignete vertrauensbildende Maßnahmen sind nötig. Die Bundesregierung muss deutsche Rüstungsexporte in diese Region unterbinden und dafür auch international eintreten.

Es steht zu befürchten, dass der von der UNO gegenwärtig verfolgte und von den USA argwöhnisch beäugte diplomatische Lösungsweg letztendlich torpediert werden wird. Mit dem dann folgenden Krieg wächst die große Gefahr, dass die gesamte Region, die ohnehin schon gravierend unter dem Nahostkonflikt leidet, in Brand gesetzt wird.

Gerade deshalb wäre europäische Politik gefragt: Die zivile »europäische Handschrift« muss erkennbar werden. Die Geschichte beweist doch, dass die Europäer immer dann eine friedensstiftende Rolle spielten und dabei selbst gewannen, wenn sie sich auf ihre grundlegenden Interessen besannen und sie selbstbewusst wahrnahmen. Auf verschiedene Weise haben solch große europäische Politiker wie Willy Brandt, Olof Palme und Charles de Gaulle diese Eigenständigkeit praktiziert. Dieses Format wird wieder benötigt.

\section{Für eine neue Qualität in den Beziehungen Deutsch- lands und der EU zu Russland}

$\mathrm{Zu}$ den besonders wichtigen und mit größter Sorgfalt zu pflegenden internationalen Beziehungen gehört aus unserer Sicht auch im 21. Jahrhundert das Verhältnis zu Russland. Aus der gemeinsamen Geschichte und Kultur, aus der geographischen Lage und der gegenseitigen Sicherheitsabhängigkeit, aus wirtschaftlichen und außenpolitischen Faktoren ergeben sich eine Reihe von strategischen Schnittmengen. Nach dem Ende des Ost-West-Konflikts wurden sie von den Bundesregierungen wie auch der EU unterschiedlich genutzt. Die aus westlicher Sicht unzulänglichen Transformationserfolge in Russland, die 
andersgearteten demokratischen Verhältnisse wurden im Westen nur zu oft und gern als Vorwand genommen, um wirkliche kooperative Beziehungen höchst sparsam zu entwickeln, wenn nicht gar zu verweigern. Auch Deutschland bequemte sich meist nur dann zur Zusammenarbeit, wenn kurzfristige außenpolitische Interessen dies geboten erscheinen ließen. Die Politikwissenschaft hat für die vergangenen zehn Jahre nicht umsonst die Formel »verlorenes Jahrzehnt « geprägt, der ich nicht gänzlich folge. Aber Tatsache ist: Charakter und Stand der Beziehungen entsprechen auch heute weder den Möglichkeiten noch den Erfordernissen. Und mit dem labilen inneren Zustand der russischen Gesellschaft lässt sich noch immer vieles entschuldigen.

Mit Präsident Putin scheint zum ersten Mal nach dem Zusammenbruch der Sowjetunion ein Führer die Lenkung des russischen Staates übernommen zu haben, der fest entschlossen ist, den entstandenen internationalen Realitäten Rechnung zu tragen und die erforderlichen Schlussfolgerungen für die russische Politik zu ziehen. Wie seine Rede vor dem Bundestag zeigte, ist er - offensichtlich aus der Lage heraus - bemüht, sich dabei alle Optionen offen zu halten - gegenüber Europa und Deutschland, gegenüber den USA ebenso wie China.

Die Bush-Administration hat nach den Terrorangriffen des 11. September betont, dass eine wirkungsvolle Antwort auf den internationalen Terrorismus, auf die Verbreitung von Massenvernichtungswaffen oder sich neu entfaltende Bedrohungen ohne stabile Beziehungen zu Russland nicht möglich wäre. ${ }^{4}$ Diese Entwicklung ist überaus ambivalent zu bewerten, sie eröffnet aber auch Chancen zu einer wirklichen Vertiefung der wechselseitigen Beziehungen. Die bisherige West-Ost-Kooperation genügt aber den Erfordernissen und Möglichkeiten nicht. Sie ist zu sehr bevormundend, zu wenig kooperativ, sie ist zu sehr auf einzelne Veranstaltungen und Projekte auf wirtschaftlichem oder kulturellem Gebiet zugeschnitten. Gerade für Deutschland gilt es, mit Phantasie, Ideenreichtum und noch mehr Aktivität die Chance zu nutzen, um zu qualitativ neuen kooperativen Beziehungen zur Russischen Föderation (wie übrigens auch zu China) zu kommen. So sollte schnellstmöglich in einem Regierungsabkommen die längerfristige Entwicklung der Wirtschaftsbeziehungen zwischen beiden Staaten in wirtschaftlich und politisch vernünftigen und profitablen Projekten vereinbart werden, wobei den neuen Bundesländern sowie der Schaffung von Arbeitsplätzen besondere Aufmerksamkeit gewidmet wird. Es sollten neue Förder- und Finanzierungsinstrumente ausgearbeitet werden, die Export-Import-Abwicklungen zwischen beiden Ländern finanziell stimulieren, Anschubfinanzierungen unbürokratisch ermöglichen und die Zusammenarbeit von Partnern beider Länder zu EU-marktüblichen Konditionen sichern. Das Angebot Putins, einen Beitrag zur Energiesicherheit Europas $\mathrm{zu}$ leisten, sollte endlich aufgegriffen und mit deutschen und europäischen Investitionen in der russischen Energiewirtschaft untersetzt werden. Die Schaffung einer DeutschRussischen Akademie als ständige Begegnungs- und gemeinsame Forschungsstätte, die Einsetzung eines RusslandBeauftragten, ein gemeinsames Langzeit-Umweltprogramm

4 Vgl. George Robertson, A New Quality in the NATO-Russia Relationship, in: International Affairs (Moskau), 1/2002, S. 32. und weitere Vorschläge zum Austausch auf vielen Gebieten wären ebenso zu nennen.

Eine solch umfassende Intensivierung der deutsch-russischen Beziehungen wäre auch die geeignete Basis, um die gebotene Kritik an dunklen Seiten der russischen Politik, sprich vor allem dem Tschetschenien-Krieg, anbringen zu können.

\section{Eine moderne Außenpolitik braucht Öffentlichkeit}

In meinen Augen ist Außenpolitik zu wichtig, um sie der Regierung allein zu überlassen. Sie wirft grundlegende Fragen nach dem Staat und seinem internationalen Handeln auf Fragen, die ganz wesentlich über die Sicherheit, aber auch das Wohlergehen der Menschen in unserem Lande entscheiden. Deshalb kann die öffentliche Diskussion über eine moderne Außenpolitik unseres Landes gar nicht intensiv genug geführt werden. Außerdem sind die meisten Menschen das Gerede von den angeblichen oder vermeintlichen Sachzwängen leid, die keine Alternativen zuließen. Moderne Außenpolitik muss deshalb gerade die Rückkopplung, den Dialog mit dem Volk suchen und nicht ängstlich darüber wachen, wie Außenminister Fischer formuliert hat, dass »der Regierungseinfluss « nicht beeinträchtigt werde. Ganze Seiten ließen sich mit Beispielen füllen, wie gerade die rotgrüne Koalition mit allen Mitteln versucht, diesem Dialog auszuweichen bzw. ihn ganz zu verhindern. Außenpolitik muss aufhören, »Geheimwissenschaft « zu sein. Die Lösung der schwierigen Fragen der Außen-, Friedens-, Sicherheits- und Entwicklungspolitik braucht den öffentlich-wissenschaftlichen Diskurs. Mehrere Friedensforschungsinstitute und noch mehr Friedensforscher liefern dazu auch in Deutschland immer wieder Anstöße und Ideen, während die Bundesregierung sich weitgehend als »beratungsresistent « erweist. Eine moderne deutsche Außenpolitik würde auch der aktiven Mitarbeit der zahlreichen NGOs nicht nur verbal einen besonderen Platz einräumen, sondern sie als unersetzbare und wichtige Partner akzeptieren.

Die PDS will eine moderne deutsche und europäische Außenpolitik, die sich selbstbewusst am Ziel eines gerechten Friedens in einer neuen, solidarischen Weltordnung orientiert. Wir dürfen dabei nicht auf einen »Reformpräsidenten $\aleph^{5}$ in der einzig verbliebenen Supermacht warten. Die Courage für einen Paradigmenwechsel in der internationalen Politik müssen die europäischen Staaten schon allein aufbringen. Das Projekt »Zivilmacht Europa«, in enger deutsch-französischer Zusammenarbeit vorangetrieben, auf eine gleichberechtigte Zusammenarbeit mit den Ländern des »Südens« gerichtet, wäre ein Zeichen der Hoffnung in einer immer weiter vernetzten und damit krisenanfälligeren Welt. Denn es ist so: Im Zeitalter der Globalisierung entscheidet sich auch das Schicksal unseres Volkes wie vieler Völker letztlich in der Außenpolitik. Das zu vergessen, wäre ein unverzeihlicher Fehler!

5 Oskar Lafontaine, Die Wut wächst. Politik braucht Prinzipien, München 2002, S. 264. 